DOE/ER/40762-252

UMD-PP\#02-043

\title{
Chiral structure of nucleon gravitational form factors
}

\author{
A.V. Belitsky, X. Ji \\ Department of Physics \\ University of Maryland at College Park \\ College Park, MD 20742-4111, USA
}

\begin{abstract}
We study the low momentum behavior of nucleon gravitational form factors in the framework of the heavy baryon chiral perturbation theory. At zero recoil they determine the momentum and spin apportion between nucleon constituents. Our result provides an insight into the response of the nucleon's pion cloud to an external weak gravitational field and establishes a theoretical framework for extrapolation of experimental and lattice data on the nucleon form factors to zero momentum transfer. We also discuss form factors corresponding to higher-rank tensor currents related to the moments of generalized parton distributions.
\end{abstract}

Keywords: nucleon spin structure, chiral perturbation theory, form factors

PACS numbers: 12.39.Fe, 11.30.Rd, 14.20.Dh 
1. The fundamental problem of hadronic physics is the understanding of the structure of the nucleon, - the building block of our planet and its inhabitants, — in terms of quark and gluonic degrees of freedom. The nucleon's mass and momentum content is one of the issues being under study for decades. Higgs mechanism giving quarks their masses generates only a tiny fraction of the total nucleon mass, the bulk of which comes entirely from the complicated dynamics of colored glue. This idea was supported by deeply inelastic scattering experiments which revealed that quarks and gluons are equally responsible for the energy-momentum structure of the nucleon with both of them carrying about $50 \%$ of its momentum. A similar study has to be addressed for the spin content of hadrons. The nucleon being a composite particle builds its spin from the angular momenta円 of its constituents $J_{q, g}$, i.e., in the case of quarks from their spin $\Delta \Sigma / 2$ and orbital motion $L_{q}$,

$$
\frac{1}{2}=\frac{\Delta \Sigma}{2}+L_{q}+J_{g}
$$

A next natural question arises about the numerical apportion between these contributions. The naive quark model (QM) attributes the whole of the nucleon spin to the sum of the ones of quarks $\Delta \Sigma_{\mathrm{QM}}=1$ [自]. However, the experimental data tells the opposite: only a small fraction of the total spin is carried by quarks $\Delta \Sigma_{\exp }=0.16 \pm 0.08$ [2]. Thus in order to test the spin sum rule (1) one has to have access to the other sources of the nucleon spin $L_{q}$ and $J_{g}$. The nucleon's angular momentum is expressed in QCD by a hadronic matrix element of the coordinate-space moment of the quark and gluon momentum densities given by specific components of the energymomentum tensor. At this point recall that the magnetic moment of a particle is the space moment of the electric current flow and can be measured as a matrix element of the electric current at non-zero momentum transfer, i.e., in elastic electron-nucleon scattering by accessing electromagnetic form factors. The same methodology is applicable in the current circumstances so that the missing orbital momentum parts can be deduced from off-forward matrix elements of the QCD energy-momentum tensor. Thus, in order to understand the nucleon spin content one has to study nucleon gravitational form factors. These functions, which arise in the nucleon

\footnotetext{
${ }^{1}$ For a gauge particle the decomposition of its angular momentum into its spin and orbital components is not possible. The hand-waiving argument goes as follows: In quantum mechanics, the wave function of a spin-s particle is a symmetric rank- $2 s$ spinor having $(2 s+1)$ components which transform into each other under the rotation of the coordinate system. The orbital wave function is related to the coordinate dependence of wave functions and is given by the spherical harmonics of order $l$ for the angular momentum $l$ of the system. Therefore, in order to distinguish clearly between the spin and orbital momentum, the spin and coordinate properties must be independent. As it is obvious, this condition is not fulfilled for gauge particles whose description in terms of field operators inevitably involves a gauge condition. For instance, in the Coulomb gauge the gluon wave function is given by the three-potential $\boldsymbol{A}^{a}(x)$ equivalent to the second rank spinor which, however, is a subject for the gauge condition $\operatorname{div} \boldsymbol{A}^{a}(x)=0$. As a result the coordinate dependence of the vector cannot be independently defined for each of its components and leads to the inability to separate the spin and orbital degrees of freedom.
} 
scattering off a weak gravitational field, are measurable experimentally in exclusive processes such as deeply virtual Compton scattering [3, 4, 5] and hard exclusive meson production [6, 7]. Moreover, they can be simulated on a lattice. As we just said, apart from the interest in their own right, gravitational form factors at zero recoil determine the quark and gluon contributions to the angular momentum. In practical determination of the latter either in laboratory or lattice experiments one needs in all cases to perform an extrapolation to the limit of zero momentum transfer $\Delta^{2}=0$ to get the net effect. The experimental kinematics is restricted to low $t$-channel momenta of order $0.1 \mathrm{GeV}^{2}$ [5] and, thus, favors the applicability of the chiral perturbation theory $(\chi \mathrm{PT})$ [8] to unravel the small-momentum transfer behavior of the form factors in question. On a lattice, the minimal achieved momentum is limited by the size of the lattice. The first Monte Carlo simulations [9, 10] were done with lattices $16^{2} \times 32$ where $\Delta_{\min }^{2} \sim 0.4 \mathrm{GeV}^{2}$. Thus in order to enter the regime of validity of $\chi \mathrm{PT}$ one needs repeat the simulations with bigger lattices. In the present Letter we compute one-loop contributions to off-forward matrix elements of local quark and gluon composite operators and thus determine their momentum transfer dependence.

2. The spin and momentum structure of the nucleon is carried by off-forward matrix elements of quark and gluon composite operators. In our consequent presentation we will be concerned with the parity-even sector which can shed some light on the spin structure of the nucleon. Under chiral $S U(2)_{L} \otimes S U(2)_{R}$ group the operators transform either as isovector $(3,1) \oplus(1,3)$ or isoscalar $(1,1)$ representations and correspond to flavor non-singlet $\mathcal{R}^{q, a}$ and singlet $\mathcal{R}^{q, 0}$ quark operators, respectively,

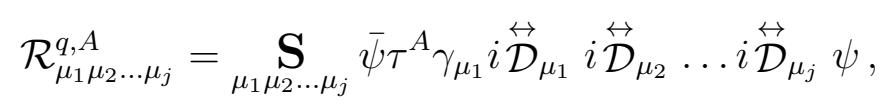

where $\tau^{A}=\left(\mathbb{1}, \tau^{a}\right)$ with $\mathbb{1} \equiv 1_{[2] \times[2]}$. The operator of symmetrization and trace subtraction is defined as follows, e.g., $\mathbf{S}_{\mu_{1} \mu_{2}} t_{\mu_{1} \mu_{2}}=\frac{1}{2 !}\left(t_{\mu_{1} \mu_{2}}+t_{\mu_{2} \mu_{1}}-\frac{1}{2} g_{\mu_{1} \mu_{2}} t_{\mu_{\mu}}\right)$. The isoscalar combination $\mathcal{R}^{q, 0}$ mixes under the renormalization group evolution with gluonic operators,

$$
\mathcal{R}_{\mu_{1} \mu_{2} \ldots \mu_{j}}^{g}=\underset{\mu_{1} \mu_{2} \ldots \mu_{j}}{\mathbf{S}} G_{\nu \mu_{1}} i \overleftrightarrow{\mathcal{D}}_{\mu_{1}} i \stackrel{\leftrightarrow}{\mathcal{D}}_{\mu_{2}} \ldots i \stackrel{\leftrightarrow}{\mathcal{D}}_{\mu_{j-1}} G_{\mu_{j} \nu}
$$

The nucleon matrix element of these operators are parametrized via 'form factors' as follows

$$
\begin{aligned}
\left\langle p_{2}\left|\mathcal{R}_{\mu_{1} \ldots \mu_{j}}^{A}\right| p_{1}\right\rangle & =\underset{\mu_{1} \ldots \mu_{j}}{\mathbf{S}} \bar{u}\left(p_{2}\right) \tau^{A} \gamma_{\mu_{1}} u\left(p_{1}\right)\left\{p_{\mu_{2}} \ldots p_{\mu_{j}} A_{j, j}\left(\Delta^{2}\right)+\ldots+\Delta_{\mu_{2}} \ldots \Delta_{\mu_{j}} A_{j, 1}\left(\Delta^{2}\right)\right\} \\
& +\underset{\mu_{1} \ldots \mu_{j}}{\mathbf{S}} \bar{u}\left(p_{2}\right) \tau^{A} \frac{i \sigma_{\mu_{1} \nu} \Delta_{\nu}}{2 M} u\left(p_{1}\right)\left\{p_{\mu_{2}} \ldots p_{\mu_{j}} B_{j, j}\left(\Delta^{2}\right)+\ldots+\Delta_{\mu_{2}} \ldots \Delta_{\mu_{j}} B_{j, 1}\left(\Delta^{2}\right)\right\} \\
& +\underset{\mu_{1} \ldots \mu_{j}}{\mathbf{S}} \frac{\bar{u}\left(p_{2}\right) \tau^{A} u\left(p_{1}\right)}{2 M} \Delta_{\mu_{1}} \ldots \Delta_{\mu_{j}} C_{j}\left(\Delta^{2}\right),
\end{aligned}
$$

where we have introduced the vectors $p \equiv p_{1}+p_{2}$ and $\Delta=p_{2}-p_{1}$ and assumed the normalization for hadronic states $\langle p \mid p\rangle=2 p_{0} V$ and bispinors $\bar{u} u=2 M$. We have reduced in the above equation all tensor structures of the scalar Dirac bilinear containing at least one vector $p_{\mu}$ by means of 
the Gordon identity to the vector and tensor Dirac bilinears of the first two lines. There is an important constraints on the matrix elements (四) imposed by the time-reversal invariance, namely, vanishing of Lorentz structures with odd powers of the momentum transfer $\Delta$ [3]. We will keep this condition in mind without implementing it explicitly in our subsequent considerations.

The reduced matrix elements $A, B$ and $C$ are related to the moments of parity-even quark or gluon generalized parton distributions [11, 12, 4] measurable in deeply virtual Compton scattering via

$$
\begin{aligned}
\int_{-1}^{1} d x x^{j-1} H\left(x, \eta, \Delta^{2}\right) & =\sum_{k=0}^{j-1} \eta^{k} A_{j, j-k}\left(\Delta^{2}\right)+\eta^{j} C_{j}\left(\Delta^{2}\right), \\
\int_{-1}^{1} d x x^{j-1} E\left(x, \eta, \Delta^{2}\right) & =\sum_{k=0}^{j-1} \eta^{k} B_{j, j-k}\left(\Delta^{2}\right)-\eta^{j} C_{j}\left(\Delta^{2}\right) .
\end{aligned}
$$

The angular momentum sum rule (11) involves the form factors at zero recoil entering their second moment [12]

$$
J=\frac{1}{2} \lim _{\Delta^{2} \rightarrow 0}\left\{A_{2,2}\left(\Delta^{2}\right)+B_{2,2}\left(\Delta^{2}\right)\right\},
$$

which are attributed to quarks or gluons depending on the contributing composite operators.

We consider single-nucleon systems and, in order to have a consistent power counting, use the formalism of the heavy baryon $\chi \mathrm{PT}$ which treats the nucleon as a non-relativistic infinitely heavy particle [13]. For the purpose of a unique translation of the Lorentz-covariant matrix elements in Eq. (4) into non-relativistic ones its is convenient to use the Breit reference frame [14, 15]. It is defined by the condition $\boldsymbol{p}=0$ which leads in turn to $\Delta_{0}=0, p_{0}=2 M \sqrt{1-\Delta^{2} /\left(4 M^{2}\right)} \equiv 2 M \delta$, and $\boldsymbol{p}_{2}=-\boldsymbol{p}_{1}=\boldsymbol{\Delta} / 2$. In the Breit frame, the Dirac bilinears arising in Eq. (田) are reduced via equations

$$
\begin{aligned}
& \bar{u}\left(p_{2}\right) \gamma_{\mu} u\left(p_{1}\right)=v_{\mu} \bar{u}_{v}\left(p_{2}\right) u_{v}\left(p_{1}\right)+\frac{1}{M} \bar{u}_{v}\left(p_{2}\right)\left[S_{\mu}, S \cdot \Delta\right] u_{v}\left(p_{1}\right), \\
& \bar{u}\left(p_{2}\right) \frac{i \sigma_{\mu \nu} \Delta_{\nu}}{2 M} u\left(p_{1}\right)=v_{\mu} \frac{\Delta^{2}}{4 M^{2}} \bar{u}_{v}\left(p_{2}\right) u_{v}\left(p_{1}\right)+\frac{1}{M} \bar{u}_{v}\left(p_{2}\right)\left[S_{\mu}, S \cdot \Delta\right] u_{v}\left(p_{1}\right),
\end{aligned}
$$

to the ones constructed from the projected large components of the heavy baryon bispinor $u_{v}\left(p_{i}\right) \equiv$ $(1+\not) u\left(p_{i}\right) / \sqrt{2+2\left(v \cdot p_{i}\right) / M}$ normalized as $\bar{u}_{v} u_{v}=2 M$. The velocity $v$ arises in the heavy-mass decomposition of the incoming and outgoing nucleon momenta $p_{1,2}=M v+k \mp \Delta / 2$ and the residual momentum is defined as $k=(M(\delta-1), \mathbf{0})$ when $v=(1, \mathbf{0})$. The spin vector $S_{\mu} \equiv \frac{i}{2} \sigma_{\mu \nu} \gamma_{5} v_{\nu}$ reduces to the spin three-vector $S=(0, \boldsymbol{\Sigma} / 2)$ in the nucleon rest frame. Exploiting these results, the decomposition (4) transforms in the Breit frame to the equation

$$
\begin{aligned}
& \left\langle p_{2}\left|\mathcal{R}_{\mu_{1} \ldots \mu_{j}}^{A}\right| p_{1}\right\rangle=\underset{\mu_{1} \ldots \mu_{j}}{\mathbf{S}} \bar{u}_{v}\left(p_{2}\right) \tau^{A} u_{v}\left(p_{1}\right) v_{\mu_{1}}\left\{(2 M \delta)^{j-1} E_{j, j}\left(\Delta^{2}\right) v_{\mu_{2}} \ldots v_{\mu_{j}}+\ldots+E_{j, 1}\left(\Delta^{2}\right) \Delta_{\mu_{2}} \ldots \Delta_{\mu_{j}}\right\} \\
& \quad+\underset{\mu_{1} \ldots \mu_{j}}{\mathbf{S}} \frac{1}{M} \bar{u}_{v}\left(p_{2}\right) \tau^{A}\left[S_{\mu_{1}}, S \cdot \Delta\right] u_{v}\left(p_{1}\right)\left\{(2 M \delta)^{j-1} M_{j, j}\left(\Delta^{2}\right) v_{\mu_{2}} \ldots v_{\mu_{j}}+\ldots+M_{j, 1}\left(\Delta^{2}\right) \Delta_{\mu_{2}} \ldots \Delta_{\mu_{j}}\right\}
\end{aligned}
$$




$$
+\underset{\mu_{1} \ldots \mu_{j}}{\mathbf{S}} \frac{\bar{u}_{v}\left(p_{2}\right) \tau^{A} u_{v}\left(p_{1}\right)}{2 M \delta^{-1}} \Delta_{\mu_{1}} \ldots \Delta_{\mu_{j}} C_{j}
$$

where we have introduced a short-hand notation for the generalized electric- and magnetic-like form factors

$$
E_{j, k} \equiv A_{j, k}+\frac{\Delta^{2}}{4 M^{2}} B_{j, k}, \quad M_{j, k} \equiv A_{j, k}+B_{j, k}
$$

Now we are in a position to address the calculation of the small- $\Delta$ dependence of these form factors. As it is obvious from the tensor decomposition, one cannot compute form factors accompanying Lorentz tensors involving several $\Delta_{\mu}$, i.e., $k<j-2$, while restricting oneself to next-to-leading order in the chiral expansion. Also note that $E_{1,1}$ and $M_{1,1}$ correspond to the standard Sachs nucleon electromagnetic form factors extensively discussed in the literature [14, 15] and, therefore, their evaluation will not be repeated presently.

3. The calculational procedure is rather straightforward. First, one constructs composite operators from the nucleon and pion fields which match the quantum numbers of the ones on the quark-gluon level (2, 3) and adds them to the effective Lagrangian [16, 17]. Then one uses the heavy-baryon chiral perturbation theory [13], see also the review [18, for the computation of tree and one-loop contributions. For the case at hand, one needs the leading order pion-nucleon Lagrangian

$$
\mathcal{L}=\bar{N}_{v}\left\{i v \cdot \mathcal{D}+2 g_{A} S \cdot \mathcal{A}\right\} N_{v}+\frac{f_{\pi}^{2}}{4} \operatorname{tr}\left\{\partial_{\mu} \Sigma \partial_{\mu} \Sigma^{\dagger}\right\}+\lambda \operatorname{tr}\left\{M_{q}\left(\Sigma+\Sigma^{\dagger}\right)\right\}
$$

The axial-vector and vector (in the covariant derivative $\mathcal{D}_{\mu} \equiv \partial_{\mu}+\mathcal{V}_{\mu}$ ) pion potentials are

$$
\mathcal{V}_{\mu} \equiv \frac{1}{2}\left(\xi^{\dagger} \partial_{\mu} \xi+\xi \partial_{\mu} \xi^{\dagger}\right), \quad \mathcal{A}_{\mu} \equiv \frac{i}{2}\left(\xi^{\dagger} \partial_{\mu} \xi-\xi \partial_{\mu} \xi^{\dagger}\right)
$$

Here $\Sigma$ is constructed from the multiplet of the pion fields

$$
\Sigma \equiv \xi^{2}=\exp \left(\frac{i}{f_{\pi}} \vec{\pi} \cdot \vec{\tau}\right)
$$

and $f_{\pi}=93 \mathrm{MeV}$ the pion decay constant. Expanding to the first non-trivial order in the pion fields we get for the axial and vector potentials: $\mathcal{A}_{\mu}=-\frac{1}{2 f_{\pi}} \partial_{\mu} \vec{\pi} \cdot \vec{\tau}+\ldots$ and $\mathcal{V}_{\mu}=$ $\frac{i}{\left(2 f_{\pi}\right)^{2}} \varepsilon_{a b c} \tau^{c} \pi^{a} \partial_{\mu} \pi^{b}+\ldots$, respectively. In the mass term, the coefficient $\lambda$ is related to the quark condensate via $\lambda=-\frac{1}{2}\langle\bar{\psi} \psi\rangle$ and the quark mass matrix is $M_{q}=\operatorname{diag}\left(m_{u}, m_{d}\right)$.

Ultraviolet divergences generated by one-loop diagrams have to be absorbed into coefficients of next-to-leading order counterterms in the chiral expansion.

Now we are in a position to discuss the construction of a basis of hadronic twist-two operators corresponding to the quark and gluon ones in Eqs. (2) and (3). There are two types of local hadronic operators: built either from the pions alone or bilinear in the nucleon fields. The nucleon 
operators have the form which mimics the tensor decomposition of the off-forward matrix elements. The leading operators contributing to isoscalar and isovector combinations of $E_{j, j}$ and $M_{j, j}$ are

$$
\begin{aligned}
\mathcal{O}_{\mu_{1} \mu_{2} \ldots \mu_{j}}^{N, A} & =a_{j}^{N}(2 M)^{j-1} \underset{\mu_{1} \ldots \mu_{j}}{\mathbf{S}} v_{\mu_{1}} \ldots v_{\mu_{j}} \bar{N}_{v} \tau_{\xi+}^{A} N_{v} \\
& +b_{j}^{N}(2 M)^{j-1}\left(-i \partial_{\nu}\right) \underset{\mu_{1} \ldots \mu_{j}}{\mathbf{S}} v_{\mu_{1}} \ldots v_{\mu_{j-1}} \bar{N}_{v} \tau_{\xi+}^{A} \frac{\left[S_{\mu_{j}}, S_{\nu}\right]}{M} N_{v}+\ldots
\end{aligned}
$$

where

$$
\tau_{\xi+}^{A} \equiv \frac{1}{2}\left(\xi \tau^{A} \xi^{\dagger}+\xi^{\dagger} \tau^{A} \xi\right)
$$

The matching coefficients $a^{N}$ and $b^{N}$ from the partonic to the hadronic level are unknown and have to be determined from experimental data. Subleading operators are deduced from this expression by a mere replacement of $v$ s in the Lorentz structure by the derivatives. However, due to the time-reversal symmetry restrictions one has to replace an even number of velocities. Here and everywhere later, the nucleon operators are normalized as follows $N_{v}(0)|p\rangle=u_{v}(p)|0\rangle$.

The pion sector has to be considered separately for the isoscalar and isovector components. As we will see momentarily, one can essentially reduce the number of operators at each level in the derivative expansion provided one uses all underlying symmetries. A number of relations arises on the basis of the following 'magic' property of the Pauli matrices $\tau^{2} \vec{\tau} \tau^{2}=-\vec{\tau}^{T}$ which results into a useful conjugation property for the nonlinear pion field $\Sigma$,

$$
\tau^{2} \Sigma^{\dagger} \tau^{2}=\Sigma^{T}
$$

4. Let us start from the isoscalar sector which transforms as $(1,1)$ under chiral $S U(2)_{L} \otimes$ $S U(2)_{R}$. The leading nucleon operators are displayed in Eq. (12) for $A=0$ so that $\tau_{\xi+}^{0}=\mathbb{1}$. Next, one can immediately conclude that the lowest local operator in Eq. (2) does not correspond to any pion operator as a consequence of the unitarity of $\Sigma$ and Eq. (14),

$$
\operatorname{tr}\left\{\Sigma \partial_{\mu} \Sigma^{\dagger}\right\}=0
$$

Its generalization for an arbitrary odd $j=2 k+1$ reads

$$
\underset{\mu_{1} \mu_{2} \ldots \mu_{j}}{\mathbf{S}} \operatorname{tr}\left\{\partial_{\mu_{1}} \ldots \partial_{\mu_{k}} \Sigma \partial_{\mu_{k+1}} \ldots \partial_{\mu_{j}} \Sigma^{\dagger}\right\}=\frac{1}{2} \underset{\mu_{1} \mu_{2} \ldots \mu_{j}}{\mathbf{S}} \partial_{\mu_{1}} \operatorname{tr}\left\{\partial_{\mu_{2}} \ldots \partial_{\mu_{k+1}} \Sigma \partial_{\mu_{k+2}} \ldots \partial_{\mu_{j}} \Sigma^{\dagger}\right\}
$$

Therefore, all pion operators with an odd number of derivatives $j=2 k+1$ can be reduced to the ones containing an odd number of total derivatives, so that we have

$$
\underset{\mu_{1} \ldots \mu_{j}}{\mathbf{S}_{j}}\left\{\sum_{l=0}^{k-1} a_{j, 2 l+1}^{\pi}(-i \partial)^{2 l+1} \operatorname{tr}\left\{(-i \partial)^{k-l} \Sigma(i \partial)^{k-l} \Sigma^{\dagger}\right\}\right\}_{\mu_{1} \ldots \mu_{j}}
$$

Here, on the right-hand side of the equation, we imply that the Lorentz indices are attached to the partial derivatives $\partial$ in a particular order, e.g., $\left\{\partial^{3}\right\}_{\mu_{1} \mu_{2} \mu_{3}}=\partial_{\mu_{1}} \partial_{\mu_{2}} \partial_{\mu_{3}}$. Note, however, that 
matrix elements of these operators violate the time reversal properties of the off-forward matrix elements in question (田) and thus cannot contribute.

Using the symmetry properties established above we can easily write composite pion operators for even $j=2 k$,

$$
\mathcal{O}_{\mu_{1} \mu_{2} \ldots \mu_{j}}^{\pi}=f_{\pi}^{2} \underset{\mu_{1} \ldots \mu_{j}}{\mathbf{S}}\left\{\sum_{l=0}^{k-1} a_{j, 2 l}^{\pi}(-i \partial)^{2 l} \operatorname{tr}\left\{(-i \partial)^{k-l} \Sigma(i \partial)^{k-l} \Sigma^{\dagger}\right\}\right\}_{\mu_{1} \ldots \mu_{j}}
$$

Since quantum numbers of hadronic operators match both quark and gluon ones in Eqs. (2) and (3), the difference between the latter on the hadronic level arises only in the value of the matching coefficients which appear in two species, $a_{j, k}^{\pi, q}$ and $a_{j, k}^{\pi, g}$. Below, the index which attributes hadronic operators either to the quark or gluon sector will not be displayed explicitly for brevity. Note that $a_{j, 0}^{\pi} \equiv a_{j}^{\pi}$ survive in the forward matrix elements and, thus, are measurable in conventional deeply inelastic scattering experiments. For instance, $a_{2}^{\pi}$ is related to the momentum fraction of the pion carried by quarks and gluons forming it. For an on-shell pion at low normalization point $\mu^{2} \approx 0.3 \mathrm{GeV}^{2}$ one has $19 a_{2}^{\pi, q}=\left\langle x_{q}\right\rangle_{\pi} \approx 0.7$ and respectively $a_{2}^{\pi, g}=\left\langle x_{g}\right\rangle_{\pi} \approx 0.3$ due to the momentum conservation. There is a number of pion operators with more than two $\Sigma$ fields since their insertion does not alter the twist of the composite operator, however, they do not contribute to the one-loop matrix elements we are interested in and will be totally omitted.

To summarize, the isoscalar nucleon gravitational form factors, - the second moment of the generalized parton distributions $H$ and $E$, - receive contributions from the following pion and nucleon operators:

$$
\begin{aligned}
\mathcal{O}_{\mu_{1} \mu_{2}}^{\pi} & =f_{\pi}^{2} a_{2}^{\pi} \underset{\mu_{1} \mu_{2}}{\mathbf{S}} \operatorname{tr}\left\{\partial_{\mu_{1}} \Sigma \partial_{\mu_{2}} \Sigma^{\dagger}\right\} \\
\mathcal{O}_{\mu_{1} \mu_{2}}^{N} & =a_{2}^{N}(2 M) \underset{\mu_{1} \mu_{2}}{\mathbf{S}} v_{\mu_{1}} v_{\mu_{2}} \bar{N}_{v} N_{v}+b_{2}^{N}(2 M)\left(-i \partial_{\nu}\right) \underset{\mu_{1} \mu_{2}}{\mathbf{S}} v_{\mu_{1}} \bar{N}_{v} \frac{\left[S_{\mu_{2}}, S_{\nu}\right]}{M} N_{v} \\
& +c_{2}^{N} \frac{1}{2 M} \underset{\mu_{1} \mu_{2}}{\mathbf{S}}\left(-i \partial_{\mu_{1}}\right)\left(-i \partial_{\mu_{2}}\right) \bar{N}_{v} N_{v},
\end{aligned}
$$

and we have also included an operator with two total derivatives which generates the structure $C_{2}\left(\Delta^{2}\right)$.

A calculation of the diagrams (a) and (c), (b) and (d) do not contribute for isoscalar operators, gives

$$
\begin{aligned}
E_{2,2}\left(\Delta^{2}\right) & =a_{2}^{N}+3 a_{2}^{\pi} \frac{g_{A}^{2}}{64 \pi f_{\pi}^{2} M}\left\{\left(2 m^{2}-\Delta^{2}\right) \int_{0}^{1} d x \sqrt{m^{2}(x)}+\frac{4}{3} m^{3}\right\} \\
M_{2,2}\left(\Delta^{2}\right) & =b_{2}^{N}+3 \frac{g_{A}^{2}}{\left(4 \pi f_{\pi}\right)^{2}} \int_{0}^{1} d x\left(a_{2}^{\pi} m^{2}(x)-b_{2}^{N} m^{2} \delta(x)\right)\left\{\ln \frac{m^{2}(x)}{\Lambda_{\chi}^{2}}-1\right\} \\
C_{2}\left(\Delta^{2}\right) & =c_{2}^{N}+3 a_{2}^{\pi} \frac{g_{A}^{2}}{16 \pi f_{\pi}^{2}} M\left(2 m^{2}-\Delta^{2}\right) \int_{0}^{1} d x \frac{x(1-x)}{\sqrt{m^{2}(x)}}
\end{aligned}
$$




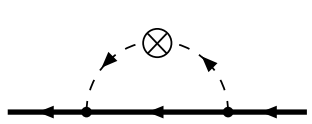

(a)

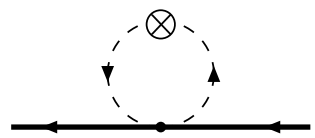

(b)

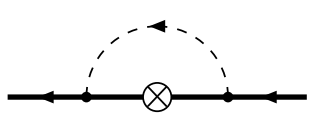

(c)

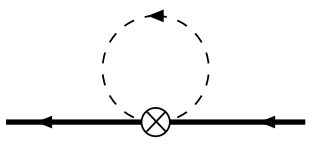

(d)

Figure 1: One loop diagrams contributing to the matrix elements of the twist-two operators. The self-energy insertions into the external line, which are not displayed explicitly, have to be added.

where $m^{2}(x) \equiv m^{2}-x(1-x) \Delta^{2}$. Note the absence of one-loop contributions to the $E$ and $C$ form factors from the nucleon operators. It is a mere consequence of a cancellation of the one-loop contribution (c) by self-energy insertions into the external lines. We have minimally absorbed ultraviolet divergences generated in $M_{2,2}$ into higher order counterterms that results into a redefinition of their coefficients, with which they enter in the effective Lagrangian $r_{i} \rightarrow$ $r_{i}+$ coeff $L_{\varepsilon}$, with $L_{\varepsilon} \equiv \frac{1}{\varepsilon}-\gamma_{E}+\ln 4 \pi$. So that the right-hand side of $M_{2,2}$ has extra analytic terms

$$
\frac{m^{2}}{\left(4 \pi f_{\pi}\right)^{2}} r_{1}+\frac{\Delta^{2}}{\left(4 \pi f_{\pi}\right)^{2}} r_{2} .
$$

Note, that one can also completely absorb analytical contributions present in (20) into counterterms. This simply sets the renormalization scheme prescription which has to be used for all observables once $r_{i}$ are fitted to experimental data in one.

Another comment concerns $E_{2,2}$. Pion operators generate $1 / M$-suppressed contributions to the structure of interest. Therefore, by power counting we have to add $\mathcal{O}(1 / M)$ bilinear nucleon operators constructed from one large and one small components of the nucleon field as well as analogous terms stemming from the chiral Lagrangian. Lorentz invariance fixes unambiguously their coefficients and no new low energy constants arise. However, the effect of these contributions in form factors is $\Delta^{2}$-independent and analytic in the pion mass. Thus they will not computed by us presently, although can be anticipated to generate an addendum $-5 a_{2}^{N} g_{A}^{2} m^{3} /\left(32 \pi f_{\pi}^{2} M\right)$ on the right-hand side of $E_{2,2}$ in Eq. (20) required by momentum sum rules discussed below. Also, there arises a Foldy-like term $\left(b_{j}^{N}-a_{j}^{N}\right) \Delta^{2} /\left(4 M^{2}\right)$ in $E_{j, j}$, similarly to the form factor case [15], from contributions of the small nucleon field components in the heavy-mass expansion of relativistic nucleon operators.

Sum rules for the total momentum and spin of the nucleon (pion) impose constraints on the coefficients $a_{2}^{N}$ and $b_{2}^{N}\left(a_{2}^{\pi}\right)$,

$$
a_{2}^{N, q}+a_{2}^{N, g}=1, \quad b_{2}^{N, q}+b_{2}^{N, g}=1, \quad a_{2}^{\pi, q}+a_{2}^{\pi, g}=1 .
$$


The latter two equations imply that the total gravitomagnetic moment of the nucleon vanishes, i.e., $B_{2,2}^{q}(0)+B_{2,2}^{g}(0)=0$ [20, 21].

The leading $(j, j)$-structures of the higher $j$-moments $(j>2)$ do not receive non-analytic contributions in the momentum transfer at next-to-leading order in the chiral expansion due to the absence of relevant pion operators. Thus one gets

$$
E_{j, j}\left(\Delta^{2}\right)=a_{j}^{N}+\ldots, \quad M_{j, j}\left(\Delta^{2}\right)=b_{j}^{N}\left(1-3 \frac{g_{A}^{2} m^{2}}{\left(4 \pi f_{\pi}\right)^{2}} \ln \frac{m^{2}}{\Lambda_{\chi}^{2}}+\ldots\right),
$$

where ellipsis stand for, at least, $\mathrm{m}^{2} /\left(4 \pi f_{\pi}\right)^{2}$-suppressed analytic contributions from one-loop diagrams and counterterms.

5. In the isovector sector, the use of Eq. (14) results into

$$
\underset{\mu_{1} \mu_{2}}{\mathbf{S}} \operatorname{tr}\left\{\tau^{a} \partial_{\mu_{1}} \Sigma \partial_{\mu_{2}} \Sigma^{\dagger}\right\}=0 \text {. }
$$

Its generalizations for even $j=2 k$ are straightforward and read

$$
\underset{\mu_{1} \mu_{2} \ldots \mu_{j}}{\mathbf{S}} \operatorname{tr}\left\{\tau^{a} \partial_{\mu_{1}} \ldots \partial_{\mu_{k}} \Sigma \partial_{\mu_{k+1}} \ldots \partial_{\mu_{j}} \Sigma^{\dagger}\right\}=0
$$

Using this property it is easy to convince oneself that pion operators for even $j=2 k$ are reduced to the ones involving an odd number of total derivatives, translated in the momentum space to odd powers of the momentum transfer,

$$
\underset{\mu_{1} \ldots \mu_{j}}{\mathbf{S}}\left\{\sum_{l=0}^{k-1} a_{2 l}^{\pi ;(j)}(-i \partial)^{2 l+1} \operatorname{tr} \tau^{a}\left\{(-i \partial)^{k-l} \Sigma(i \partial)^{k-l+1} \Sigma^{\dagger}+(-i \partial)^{k-l} \Sigma^{\dagger}(i \partial)^{k-l+1} \Sigma\right\}\right\}_{\mu_{1} \ldots \mu_{j}},
$$

which, therefore, cannot contribute to the matrix elements in question due to the time-reversal condition alluded to above.

For odd $j=2 k+1$, an elementary consideration along the same line as above leads to the following structure of the non-singlet pion operators

$$
\begin{aligned}
\mathcal{O}_{\mu_{1} \mu_{2} \ldots \mu_{j}}^{\pi, a} & =\frac{f_{\pi}^{2}}{2} \underset{\mu_{1} \ldots \mu_{j}}{\mathbf{S}} \\
& \times\left\{\sum_{l=0}^{k} a_{2 l+1}^{\pi ;(j)}(-i \partial)^{2 l} \operatorname{tr} \tau^{a}\left\{(-i \partial)^{k-l} \Sigma(i \partial)^{k-l+1} \Sigma^{\dagger}+(-i \partial)^{k-l} \Sigma^{\dagger}(i \partial)^{k-l+1} \Sigma\right\}\right\}_{\mu_{1} \ldots \mu_{j}} .
\end{aligned}
$$

The leading nucleon operators are given in Eq. (12) with $A=a$.

Thus, for the isovector gravitational form factors there no contributions from coupling to pions while the nucleon operators read

$$
\begin{aligned}
\mathcal{O}_{\mu_{1} \mu_{2}}^{N, a} & =a_{2}^{N}(2 M) \underset{\mu_{1} \mu_{2}}{\mathbf{S}} v_{\mu_{1}} v_{\mu_{2}} \bar{N} \tau_{\xi+}^{a} N+b_{2}^{N}(2 M)\left(-i \partial_{\nu}\right) \underset{\mu_{1} \mu_{2}}{\mathbf{S}} v_{\mu_{1}} \bar{N} \tau_{\xi+}^{a} \frac{\left[S_{\mu_{2}}, S_{\nu}\right]}{M} N \\
& +c_{2}^{N} \frac{1}{2 M} \underset{\mu_{1} \mu_{2}}{\mathbf{S}}\left(-i \partial_{\mu_{1}}\right)\left(-i \partial_{\mu_{2}}\right) \bar{N} \tau_{\xi+}^{a} N
\end{aligned}
$$


with $\tau_{\xi+}^{a} \equiv \frac{1}{2}\left(\xi \tau^{a} \xi^{\dagger}+\xi^{\dagger} \tau^{a} \xi\right)$.

Due to the absence of the pion cloud contribution at this order of $\chi \mathrm{PT}$, i.e., diagrams (a) and (b), no non-analytic dependence on the momentum transfer arises. However, the diagrams (c) and $(\mathrm{d})$ develop chiral logarithms in the pion mass of the form

$$
\begin{aligned}
& E_{2,2}\left(\Delta^{2}\right)=a_{2}^{N}\left\{1-\frac{m^{2}}{\left(4 \pi f_{\pi}\right)^{2}}\left(\left(3 g_{A}^{2}+1\right) \ln \frac{m^{2}}{\Lambda_{\chi}^{2}}-g_{A}^{2}-1\right)\right\}, \\
& M_{2,2}\left(\Delta^{2}\right)=b_{2}^{N}\left\{1-\frac{m^{2}}{\left(4 \pi f_{\pi}\right)^{2}}\left(\left(2 g_{A}^{2}+1\right) \ln \frac{m^{2}}{\Lambda_{\chi}^{2}}-1\right)\right\}, \\
& C_{2}\left(\Delta^{2}\right)=c_{2}^{N}\left\{1-\frac{m^{2}}{\left(4 \pi f_{\pi}\right)^{2}}\left(\left(3 g_{A}^{2}+1\right) \ln \frac{m^{2}}{\Lambda_{\chi}^{2}}-g_{A}^{2}-1\right)\right\} .
\end{aligned}
$$

We imply that one adds counterterms to the right-hand side of these equations linear in $m^{2}$ whose (unknown) coefficients absorb minimally the ultraviolet divergences stemming from loops, coeff $L_{\varepsilon}$, and the Foldy-like term as discussed after Eq. (20). The leading structures of the higher moments $M_{j, j}$ and $E_{j, j}$, apart from the change of an overall normalization $b_{2}^{N} \rightarrow b_{j}^{N}$ and $a_{2}^{N} \rightarrow a_{j}^{N}$, have the same dependence on the chiral logarithms as in Eq. (27).

6. In this Letter we used the framework of the heavy baryon chiral perturbation theory in oneloop approximation in order to predict the small momentum transfer dependence of the moments of generalized parton distributions. At next-to-leading order in the chiral expansion only the first two moments develop a non-analytic $\Delta^{2}$ behavior: isovector electromagnetic form factors and isosinglet gravitational form factors. Higher moments can have at most a linear $\Delta^{2}$-dependence at this order of $\chi \mathrm{PT}$.

Although, the momentum and spin sum rules suggest that the total quark and gluon anomalous gravitomagnetic moment is zero [20, 21], the gravitomagnetic radius of the nucleon does not vanish and reads

$$
\left.\left\langle r^{2}\right\rangle \equiv 6 \frac{d M_{2,2}\left(\Delta^{2}\right)}{d \Delta^{2}}\right|_{\Delta^{2}=0}=-3 a_{2}^{\pi} \frac{g_{A}^{2}}{\left(4 \pi f_{\pi}\right)^{2}}\left\{\ln \frac{m^{2}}{\Lambda_{\chi}^{2}}+1\right\}+6 \frac{r_{2}}{\left(4 \pi f_{\pi}\right)^{2}} .
$$

A pragmatic application of our present results consists of their use for extrapolation of experimental measurements of the angular momentum sum rule (6) to zero momentum transfer [5] and as a fit formula for lattice simulations of the parton angular momentum by means of the nucleon gravitational form factors [9]. Our considerations can be extended to other single-nucleon observables such as parity-odd generalized parton distributions as well as off-forward quark transversity and tensor gluon form factors.

We would like to thank J.-W. Chen and T.D. Cohen for useful discussions. This work was supported by the US Department of Energy under contract DE-FG02-93ER40762. 


\section{References}

[1] F.E. Close, An introduction to quarks and partons, Academic Press, (London, 1979).

[2] B.W. Filippone, X. Ji, Adv. Nucl. Part. Phys. 26 (2001) 1.

[3] X. Ji, J. Phys. G 24 (1998) 1181.

[4] A.V. Radyushkin, Phys. Rev. D 56 (1997) 5524.

[5] A.V. Belitsky, D. Müller, A. Kirchner, Theory of deeply virtual Compton scattering, hepph/0112108.

[6] J.C. Collins, L.L. Frankfurt, M. Strikman, Phys. Rev. D 56 (1997) 2982.

[7] K. Goeke, M.V. Polyakov, M. Vanderhaeghen, Prog. Part. Nucl. Phys. 47 (2001) 401.

[8] S. Weinberg, The quantum theory of fields: Modern applications, vol. 2, Cambridge University Press (Cambridge, 1996).

[9] N. Mathur, S.J. Dong, K.F. Liu, L. Mankiewicz, N.C. Mukhopadhyay, Phys. Rev. D 62 (2000) 114504.

[10] V. Gadiyak, X. Ji, C. Jung, A lattice study of the magnetic moment and the spin structure of the nucleon, hep-lat/0112040.

[11] D. Müller, D. Robaschik, B. Geyer, F. M. Dittes, J. Hořejši, Fortsch. Phys. 42 (1994) 101.

[12] X. Ji, Phys. Rev. Lett. 78 (1997) 610.

[13] E. Jenkins, A.V. Manohar, Phys. Lett. B 255 (1991) 558.

[14] V. Bernard, N. Kaiser, J. Kambor, U.-G. Meißner, Nucl. Phys. B 388 (1992) 315.

[15] V. Bernard, H.W. Fearing, T.R. Hemmert, U.-G. Meißner, Nucl. Phys. A 635 (1998) 121; (E) Nucl. Phys. A 642 (1998) 563.

[16] D. Arndt, M.J. Savage, Nucl. Phys. A 697 (2002) 429;

J.-W. Chen, X. Ji, Phys. Lett. B 523 (2001) 107.

[17] J.-W. Chen, X. Ji, Phys. Rev. Lett. 88 (2002) 052003.

[18] V. Bernard, N. Kaiser, U.-G. Meißner, Int. J. Mod. Phys. E 4 (1995) 193.

[19] M. Glück, E. Reya, I. Schienbein, Eur. Phys. J 10 (2000) 313. 
[20] O.V. Teryaev, Spin structure of nucleon and equivalence principle, hep-ph/9904376.

[21] S.J. Brodsky, D.S. Hwang, B.-Q. Ma, I. Schmidt, Nucl. Phys. B 593 (2001) 311. 